\title{
Test Particle Simulations of Ringed Barred Galaxies
}

\author{
P. Rautiainen and H. Salo
}

Department of Astronomy, University of Oulu, Oulu, Finland

G. Byrd, R. Buta and D. A. Crocker

Department of Physics and Astronomy, Box 870324, University of Alabama, Tuscaloosa, Alabama 35487

\section{Potential}

We have made test particle simulations of the gas component in barred galaxies. The potential consists of two parts: an axisymmetric isochrone potential

$$
\Phi_{0}=-\frac{1}{1+\sqrt{1+r^{2}}}
$$

and a bar potential $\Phi_{1}(r, \theta)=\Phi_{b}(r) \cos (2 \theta)$, where

$$
\Phi_{b}(r)=\left\{\begin{array}{cc}
-0.53 q\left[\left(\frac{r}{a}\right)^{2}-2.5\left(\frac{r}{a}\right)^{4}+2.1875\left(\frac{r}{a}\right)^{6}-0.65625\left(\frac{r}{a}\right)^{8}\right] & , r \leq a \\
-0.016563 q\left(\frac{r}{a}\right)^{-4} & , r>a,
\end{array}\right.
$$

The bar strength, $q$, and scalelength, $a$, are chosen so that the relative nonaxisymmetric force reaches its maximum, $10 \%$, at the distance which is $83 \%$ of the corotation radius. The potential and its scaling are the same as in Schwarz (1981).

\section{Gas clouds}

The gas component is simulated by 10000 inelastically colliding test particles. Integration of orbits is done with the leapfrog method. In the beginning of each time-step, a collision search is done for each cloud particle. Compared to many previous simulations with colliding clouds (e.g. Schwarz) the basic difference is that we do not use any grid for collisions: instead, exact intersection points and impact times are searched for each close particle pair. If a collision occurs, the relative velocities of colliding particles are changed as follows:

$$
v_{r}^{\prime}=-\alpha v_{r}
$$

where $v_{r}$ is the component of the relative velocity in the direction joining the two cloud particles before the collision and $v_{r}^{\prime}$ is the component afterwards. The coefficient of restitution, $\alpha$, is set to 0.2. For more details, see Salo (1991) and Byrd et al. (1994). 


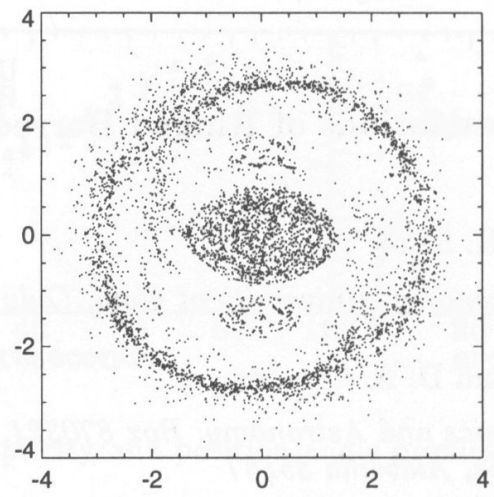

Figure 1. Particle plot for $\Omega_{b}=0.27$ after 30 bar rotations.

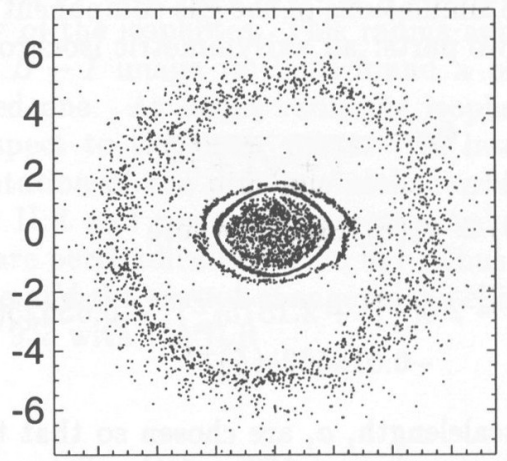

Figure 2. Particle plot for $\Omega_{b}=0.14$ after 15 bar rotations.

\section{Simulations}

When the pattern speed is high $\left(\Omega_{b}=0.27\right)$, the main resonances present are the outer Lindblad resonance (OLR) and the corotation resonance (CR). The system forms a strong $R_{2}^{\prime}$ outer pseudoring with a trace of an $R_{1}$ component, but no inner structure (see Figure 1). Buta \& Crocker (1991) illustrate an example, ESO 509-98, which may be such a fast pattern speed case.

With a medium pattern speed, $\Omega_{b}=0.14$ (see Figure 2), an inner 4:1 resonance is also present, and we get both outer and inner rings. For this particular simulation, the inner ring developed into two parts, an inner pointyoval part and an outer boxy part. No definite examples of this kind of doubled morphology have yet been identified.

When the pattern speed is slow $\left(\Omega_{b}=0.04\right)$, all the previously mentioned resonances and two inner Lindblad resonances (ILRs) are present. We get all basic ring types: outer, inner and nuclear rings. Actually two nuclear rings exist: one that is outside the inner ILR and another that is inside of it. For an example 


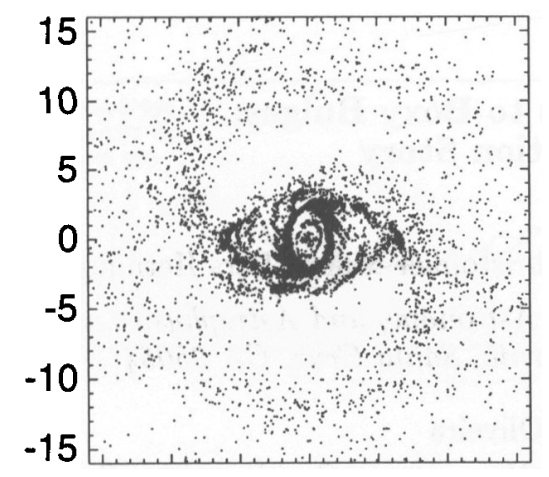

Figure 3. Particle plot for $\Omega_{b}=0.04$ after 4 bar rotations.

of a galaxy having outer, inner, and nuclear rings, see NGC 6782 in Byrd et al. (1994).

We have also studied the effect of collisions and bar strength. The features are less sharp in collisionless simulations and, for example, nuclear rings are totally missing. A stronger bar speeds up the evolution of the system. A very strong bar makes the $x_{2}$ orbit family unstable in the slow pattern speed case.

\section{Conclusions}

The test particle simulations show that each ring type is related to a certain resonance. One cannot get the ring without that resonance. The ring shapes are essentially the same as the shapes of some periodic orbits in barred potentials (see e. g. Contopoulos \& Grosbøl 1989). In addition to the pattern speed, the other two main parameters are the collision frequency and the bar strength. These results are consistent with Schwarz $(1981,1984)$.

Acknowledgments. This work was supported by the Wihuri Foundation.

\section{References}

Buta, R. \& Crocker, D. 1991, AJ, 102, 1715

Byrd, G., Rautiainen, P., Salo, H., Buta, R., \& Crocker, D.A. 1994, AJ, 108, 476

Contopoulos, G. \& Grosbøl, P. 1989, ARA\&A, 1, 261

Salo, H. 1991, A\&A, 243, 118

Schwarz, M. P. 1981, ApJ, 247, 77

Schwarz, M. P. 1984, MNRAS, 209, 93 